\title{
Updates in the Management of Early-Stage Diffuse Large B-Cell Lymphoma
}

Presented by Thomas M. Habermann, MD

\begin{abstract}
Several important updates have emerged in the management of early-stage diffuse large B-cell lymphoma. Three trials resulted in the approval of rituximab + cyclophosphamide/doxorubicin/vincristine/prednisone (R-CHOP) for use in these patients internationally. Furthermore, studies have been initiated to determine whether 4 or 6 cycles of this regimen should be administered without radiation therapy (RT). Six cycles of $\mathrm{R}$-CHOP plus central nervous system (CNS) prophylaxis and prophylactic testicular RT are recommended for patients with extranodal disease occupying the testicles. Although controversial, there is a reasonable consensus in the literature to consider 6 cycles of R-CHOP plus involved-site RT and CNS prophylaxis for patients with extranodal disease of the breast. Patients with primary bone and gastric extranodal disease do not seem to derive a significant survival benefit from RT. Molecular subtype evaluations may change treatment approaches.
\end{abstract}

J Nat Compr Canc Netw 2021;19(11.5):1324-1326 doi: 10.6004 /jnccn.2021.5108

There have been several recent updates in the management of early-stage diffuse large B-cell lymphoma (DLBCL), according to Thomas M. Habermann, MD, Professor of Medicine, Mayo Clinic College of Medicine \& Science. At the NCCN 2021 Virtual Congress: Hematologic Malignancies, he described the current treatment approach, reviewed data from recent clinical trials, and presented an individualized treatment plan integrating a PET-directed approach.

\section{DLBCL in the WHO Classification}

Based on the 2017 WHO Classification, ${ }^{1}$ there are approximately 106 types of lymphomas. Of these, 59 are mature B-cell neoplasms. Dr. Habermann noted that DLBCL-not otherwise specified occurs most frequently, with germinal center B-cell type and activated B-cell type being the two major molecular subtypes of DLBCL. "This is the histology we are primarily looking at for this particular topic," he explained. However, other histologies may come into this differential diagnosis. "It is very important to initially establish the appropriate histology."

\section{Definition}

There is no standard definition for early-stage DLBCL. However, for clinical trial eligibility, 4 main definitions have been used: Ann Arbor stage I or II; nonbulky with size ranging from $<7 \mathrm{~cm}$ to $<10 \mathrm{~cm}$; lack of B-cell symptoms; and an International Prognostic Index (IPI), age-adjusted IPI, NCCN-IPI score of 0 to 1 , or a stage-modified IPI score (removing $\geq 1$ extranodal disease and dichotomizing stage I vs II). ${ }^{2}$

To demonstrate how these definitions are implemented in clinical practice, Dr. Habermann presented the age range, bulk cutoff, extranodal disease status, and IPI score data from 4 clinical trials and 1 database registry conducted during the rituximab era (Table 1). ${ }^{3-7}$ "We have to be very careful when we try to characterize given patients through staging studies and how we want to manage them based on clinical trial data," he commented.

Further, Dr. Habermann noted that there are limited data on older patients with early-stage DLBCL. The optimal number of cycles of attenuated dosing regimens, the role of PET/CT in response-adapted therapy, and the role of radiation therapy (RT) have not been well defined in this population.

\section{Review of Data Before the Rituximab Era}

In ECOG 1484, a total of 172 patients were randomly assigned to receive 8 cycles of cyclophosphamide/doxorubicin/vincristine/prednisone $(\mathrm{CHOP}) \pm$ involved-field RT (IFRT). ${ }^{8}$ Those who underwent IFRT experienced a significantly longer duration of progression-free survival (PFS) at 6 years $(P=.05)$. According to Dr. Habermann, this was the only clinical trial that demonstrated a difference with long-term follow-up in PFS.

The CHOP regimen was developed in 1975. In 1999, it was modified by adding rituximab (R-CHOP). ${ }^{9}$ Three trials resulted in the approval of the $\mathrm{R}-\mathrm{CHOP}$ regimen internationally. ${ }^{10-12}$ "At that time and afterwards, there 


\begin{tabular}{|c|c|c|c|c|}
\hline Study & Age, y & Bulk & EN Disease & IPI \\
\hline FLYER $^{3}$ & $18-60$ & $<7.5 \mathrm{~cm}$ & $32 \%$ & aalPI, 99\% \\
\hline Optimal $>60^{4}$ & $61-80$ & $<7.5 \mathrm{~cm}$ & & IPI, 1 \\
\hline NCTN S1001 ${ }^{5}$ & $\geq 18$ & $<10 \mathrm{~cm}$ & $43 \%$ & $\begin{array}{l}\text { smIPI 0-1, } \\
69 \%\end{array}$ \\
\hline LYSA/GOELAMS $^{6}$ & $18-75$ & $<7 \mathrm{~cm}$ & $39 \%$ & $\begin{array}{l}\text { smIPI 0-1, } \\
94 \%\end{array}$ \\
\hline $\begin{array}{l}\text { British Columbia } \\
\text { Cancer Institute }\end{array}$ & 19-92 & $<10 \mathrm{~cm}$ & $52 \%$ & $\begin{array}{l}\text { smlPI 0-1, } \\
19 \%\end{array}$ \\
\hline
\end{tabular}

Abbreviations: aalPI, age-adjusted International Prognostic Index; DLBCL, diffuse large B-cell lymphoma; EN, extranodal; IPI, International Prognostic Index; smIPI, stage-modified International Prognostic Index.

were ...25 different trials that have not changed the standard of care since the initial 3 observations," Dr. Habermann commented. "No matter what we tried to do to effect change, it didn't result in improvements in overall survival (OS). R-CHOP today remains the standard of care in the upfront management of DLBCL."

\section{Where Are We in the Rituximab Era?}

"The question comes down to 4 versus 6 cycles of rituximab and CHOP without RT and PET strategies," Dr. Habermann explained. In the phase III FLYER trial of patients with aggressive B-cell lymphoma and a favorable prognosis, the PFS, event-free survival, OS, and relapse rate did not significantly differ between those who received 4 versus 6 cycles of R-CHOP. ${ }^{3}$

In the Intergroup National Clinical Trials Network Study S1001, a total of 132 eligible patients with limitedstage DLBCL underwent 3 cycles of R-CHOP therapy and an interim PET scan. ${ }^{5}$ After 4.5 years, a total of 5 patients experienced disease progression, and 2 died of lymphoma. The 5-year PFS and OS rates were $87 \%$ and $90 \%$, respectively. According to Dr. Habermann, the 5-year PFS rate was higher in patients with the germinal center B-cell subtype than in those with the activated B-cell subtype (95\% vs 70\%).

Dr. Habermann also discussed a British Colombia Cancer Lymphoid Cancer Database study of patients from March 2005 to February 2019 with limited-stage DLBCL who underwent PET/CT imaging after 3 cycles of curative-intent R-CHOP. The 5-year time to progression ( $89 \%$ vs $80 \%$ ), PFS ( $88 \%$ vs $74 \%$ ), and OS (90\% vs $77 \%$ ) rates were higher in patients who had PET-negative versus PET-positive disease." "[These results support] the use of immunochemotherapy in patients who have a negative PET scan," Dr. Habermann explained.

A retrospective, multicenter study of R-CHOP versus a more intensive immunochemotherapy regimen with or without consolidative IFRT revealed a higher complete response rate in patients with a $M Y C$ rearrangement than in those with double-hit lymphoma (98\% vs $75 \%$; $P=.003) .{ }^{13}$ Dr. Habermann noted that the complete response rate also seemed to be significantly higher in patients who underwent IFRT compared with those who did not ( $98 \%$ vs $72 \% ; P<.001)$.

\section{Scope of Extranodal DLBCL and Therapeutic Approaches}

"The IELSG-10 trial brought forward the approach of [6 cycles of] R-CHOP with central nervous system [CNS] prophylaxis and prophylactic testicular RT [for patients with extranodal early-stage DLBCL of the testicles]," Dr. Habermann commented. "There appears to be a general consensus internationally about this approach.”

There are limited data regarding the management of extranodal early-stage DLBCL occupying the breast. However, according to Dr. Habermann, 6 cycles of R-CHOP plus involved-site RT and CNS prophylaxis should be considered in this setting. "The activated B-cell phenotype predominates in early-stage DLBCL occupying the breast, and there is a risk of CNS relapse," he explained. "There is a reasonable consensus in the literature to consider this [regimen], although it is controversial." ${ }^{2,15}$

Retrospective data in the rituximab era suggest that patients with primary bone and gastric extranodal earlystage disease may not derive a significant survival benefit from RT. However, studies are ongoing for these sites. ${ }^{16,17}$

"Lastly, I think that hopefully the way to change how we approach these diseases is molecular subtype evaluation," he explained. In a 2018 article, whole-exome sequencing of 304 patients with newly diagnosed DLBCL identified 158 genetic driver alterations, and rearrangements of IGH (40\%), BCL2 (21\%), BCL6 (19\%), MYC (8\%), and PDL1/2 (5\%) were reported. ${ }^{18}$ According to Dr. Habermann, a total of 6 genetically distinct subtypes were delineated by copy number alterations and structural variations.

\section{Conclusions}

The standard of care for the initial management of patients with limited-stage DLBCL is a PET-based immunochemotherapy approach, according to Dr. Habermann. Younger patients with nonbulky stage I-II germinal center B-cell type and activated B-cell type DLBCL-not otherwise specified should be administered 4 cycles of R-CHOP if they have PET-negative disease after 2 or 3 cycles.

"IFRT is indicated in PET-positive disease in patients with a partial response after initial immunochemotherapy for early-stage DLBCL," Dr. Habermann explained. "I think the studies support that approach rather than moving to peripheral blood stem cell transplant, or even into a randomized study of a CAR T-cell therapy versus peripheral blood stem cell transplant." 
In the rituximab and PET era, the current evidence does not seem to support the routine use of RT in patients who have achieved complete remission in a nodal or extranodal mass $<7 \mathrm{~cm}$. According to Dr. Habermann, there may be exceptions in masses of the testicles, breasts, and other selected sites.

"I believe there [are] limited data on limited cycle number with or without RT in high-grade B-cell lymphoma with $M Y C, B C L 2$, or $B C L 6$ rearrangements," he explained. "I think we need more data before moving to limited cycle number."
Selected extranodal DLBCLs and different WHO histologic subtypes require more than limited therapy approaches. Dr. Habermann concluded that further studies are needed in the older patient population.

Disclosures: Dr. Habermann has disclosed serving as a consultant for Seagen, Tess Therapeutics, Lilly, MorphoSys, Incyte, BioGene, and Loxo Oncology.

Correspondence: Thomas M. Habermann, MD, Mayo Clinic Cancer Center 200 First Street SW, East 10, Rochester, MN 55905.

Email: habermann.thomas@mayo.edu

\section{References}

1. Swerdlow $\mathrm{SH}$, Campo $\mathrm{E}$, Harris NL, et al. WHO Classification of Tumours of Haematopoietic and Lymphoid Tissues. Revised 4th edition. Lyon, France: IARC Publication; 2017.

2. Zhang $X Y$, Collins GP, Cutter DJ, et al. Limited-stage diffuse large B-cell lymphoma: current management and challenges. Br J Haematol 2021 194:508-517.

3. Poeschel V, Held G, Ziepert M, et al. Four versus six cycles of CHOP chemotherapy in combination with six applications of rituximab in patients with aggressive B-cell lymphoma with favourable prognosis (FLYER): a randomised, phase 3, non-inferiority trial. Lancet 2019;394:2271-2281.

4. Pfreundschuh M, Murawski N, Christofyllaki K, et al. Excellent outcome of elderly patients with favourable-prognosis DLBCL treated with 4 cycles of CHOP/Chlip-14 plus 8 applications of rituximab and a PET-based intensification strategy the includes involved-site radiotherapy (IS-RT): results of the first 120 patients of the OPTIMAL $>60$ trial of the DSHNHL [abstract]. Blood 2017;130(Suppl 1):Abstract 1549

5. Persky DO, Li H, Stephens DM, et al. Positron emission tomography-directed therapy for patients with limited-stage diffuse large B-cell lymphoma: results of Intergroup National Clinical Trials Network Study S1001. J Clin Oncol 2020;38;3003-3011.

6. Lamy T, Damaj G, Soubeyran P, et al. R-CHOP 14 with or without radiotherapy in nonbulky imited-stage diffuse large B-cell lymphoma. Blood 2018;131:174-181

7. Sehn LH, Scott DW, Villa D, et al. Long-term follow-up of a PET-guided approach to treatment of limited-stage diffuse large B-cell lymphoma in British Columbia [abstract]. Blood 2019;134:Abstract 401.

8. Horning SJ, Weller E, Kim K, et al. Chemotherapy with or without radiotherapy in limited-stage diffuse aggressive non-Hodgkin's lymphoma: Eastern Cooperative Oncology Group Study 1484. J Clin Oncol 2004;22 3032-3038.

9. Thanarajasingam G, Minasian LM, Baron F, et al. Beyond maximum grade: modernizing the assessment and reporting in haematologic malignancies. Lancet Haematol 2018;5:e563-598.
10. Coffier B, Lepage $\mathrm{E}$, Briere J, et al. CHOP chemotherapy plus rituximab compared with $\mathrm{CHOP}$ alone in elderly patients with diffuse large B-cell Jymphoma. N Engl J Med 2002;346:235-242.

11. Habermann TM, Weller EA, Morrison VA, et al. Rituximab-CHOP versus $\mathrm{CHOP}$ alone or with maintenance rituximab in older patients with diffuse large B-cell lymphoma. J Clin Oncol 2006;24:3121-3127.

12. Pfreundschuh $M$, Trumper L, Osterborg A, et al. CHOP-like chemotherapy plus rituximab versus $\mathrm{CHOP}$-like chemotherapy alone in young patients with good prognosis diffuse large B-cell lymphoma: a randomized controlled trial by the MabThera International Trial (MInT) Group. Lancet Oncol 2006:7:379-391.

13. Torka P, Kothari SK, Sundaram S, et al. Outcomes of patients with limited-stage aggressive large B-cell lymphoma with high-risk cytogenetics. Blood Advances 2020;4:253-262

14. Vitolo U, Chiappella A, Ferreri AJM, et al. First-line treatment for primary testicular diffuse large B-cell lymphoma with rituximab-CHOP, CNS prophylaxis, and contralateral testis irradiation: final results of an international phase II trial. J Clin Oncol 2011;29:2766-2772.

15. Cheah CY, Campbell BA, Seymour JF, et al. Primary breast lymphoma Cancer Treat Rev 2014;40:900-908.

16. Hui D, Proctor B, Donaldson J, et al. Prognostic implications of extranodal involvement in patients with diffuse large B-cell lymphoma treated with rituximab and cyclophosphamide, doxorubicin, vincristine, and prednisone. Leuk Lymphoma 2010;51:1658-1667.

17. Gutiérrez-Garcia G, Colomo L, Villamor N, et al. Clinico-biological characterization and outcome of primary nodal and extranodal diffuse large Bcell lymphoma in the rituximab era. Leuk Lymphoma 2010;51: 1225-1232.

18. Chapuy B, Stewart C, Dunford AJ, et al. Molecular subtypes of diffuse large $B$ cell lymphoma are associated with distinct pathogenic mechanisms and outcomes. Nat Med 2018;24:679-690. 